\title{
CDISC SEND Domain Abbreviation Terminology
}

National Cancer Institute

\section{Source}

National Cancer Institute. CDISC SEND Domain Abbreviation Terminology. NCI

Thesaurus. Code C111113.

Terminology associated with the SEND domain abbreviation codelist of the Clinical Data Interchange Standards Consortium (CDISC) Standard for the Exchange of Non-clinical Data (SEND). 